\title{
Pathological Internet Use and Risk-Behaviors among European Adolescents
}

Tony Durkee ${ }^{1, *}$, Vladimir Carli ${ }^{1}$, Birgitta Floderus ${ }^{2}$, Camilla Wasserman ${ }^{3,4}$, Marco Sarchiapone ${ }^{3,5}$, Alan Apter ${ }^{6}$, Judit A. Balazs ${ }^{7,8}$, Julio Bobes ${ }^{9}$, Romuald Brunner ${ }^{10}$, Paul Corcoran ${ }^{11}$, Doina Cosman ${ }^{12}$, Christian Haring ${ }^{13}$, Christina W. Hoven ${ }^{4,14}$, Michael Kaess ${ }^{10}$, Jean-Pierre Kahn ${ }^{15}$, Bogdan Nemes ${ }^{12}$, Vita Postuvan ${ }^{16}$, Pilar A. Saiz ${ }^{9}$, Peeter Värnik ${ }^{17}$ and Danuta Wasserman 1

1 National Centre for Suicide Research and Prevention of Mental Ill-Health (NASP), Karolinska Institutet, Stockholm SE-17177, Sweden; vladimir.carli@ki.se (V.C.); danuta.wasserman@ki.se (D.W.)

2 Department of Clinical Neuroscience, Karolinska Institutet, Stockholm SE-17177, Sweden; birgitta.floderus@ki.se

3 Department of Medicine and Health Science, University of Molise, Campobasso 86100, Italy; camillaw@gmail.com (C.W.); marco.sarchiapone@gmail.com (M.S.)

4 Department of Child and Adolescent Psychiatry, New York State Psychiatric Institute, Columbia University, New York, NY 10032, USA; ch42@cumc.columbia.edu

5 National Institute for Migration and Poverty, Via San Gallicano, Roma 25/A, Italy

6 Feinberg Child Study Centre, Schneider Children's Medical Centre, Tel Aviv University, Tel Aviv 49202, Israel; asapter@gmail.com

7 Vadaskert Child and Adolescent Psychiatric Hospital, Budapest 1021, Hungary; judit.agnes.balazs@gmail.com

8 Institute of Psychology, Eötvös Loránd University, Budapest 1064, Hungary

9 Department of Psychiatry, Center for Biomedical Research in the Mental Health Network (CIBERSAM), University of Oviedo, Oviedo 33006, Spain; bobes@uniovi.es (J.B.); pilaralejandra.saiz@gmail.com (P.A.S.)

10 Section for Disorders of Personality Development, Clinic of Child and Adolescent Psychiatry, Centre of Psychosocial Medicine, University of Heidelberg, Heidelberg 69115, Germany; romuald.brunner@uni-heidelberg.de (R.B.); Michael.kaess@uni-heidelberg.de (M.K.)

11 National Suicide Research Foundation, Western Rd., Cork, Ireland; pcorcoran@ucc.ie

12 Department of Clinical Psychology, Iuliu Hatieganu University of Medicine and Pharmacy, Str. Victor Babes Nr. 8, Cluj-Napoca 400000, Romania; doina_octaviancosman@yahoo.com (D.C.); nemes_bogdan@yahoo.com (B.N.)

13 Research Division for Mental Health, University for Medical Information Technology (UMIT), Klagenfurt, Innsbruck 6060, Austria; Christian.haring@tilak.at

14 Department of Epidemiology, Mailman School of Public Health, Columbia University, New York, NY 10032, USA

15 Department of Psychiatry, Centre Hospitalo-Universitaire de Nancy, Université de Lorraine, Nancy, Vandoeuvre-lès-Nancy 54500, France; jp.kahn@chu-nancy.fr

16 Slovene Center for Suicide Research, Andrej Marušič Institute, University of Primorska, Koper 6000, Slovenia; vita.postuvan@upr.si

17 Centre of Behavioral and Health Sciences, Estonian-Swedish Mental Health \& Suicidology Institute, Tallinn University, Tallinn 10120, Estonia; peeterv@suicidology.ee

* Correspondence: tony.durkee@ki.se; Tel.: +46-852-486-935; Fax: +46-8-30-64-39

Academic Editor: Paul B. Tchounwou

Received: 1 December 2015; Accepted: 3 March 2016; Published: 8 March 2016

Abstract: Risk-behaviors are a major contributor to the leading causes of morbidity among adolescents and young people; however, their association with pathological Internet use (PIU) is relatively unexplored, particularly within the European context. The main objective of this study is to investigate the association between risk-behaviors and PIU in European adolescents. This cross-sectional study was conducted within the framework of the FP7 European Union project: Saving and Empowering Young Lives in Europe (SEYLE). Data on adolescents were collected from randomized schools within study sites across eleven European countries. PIU was measured using Young's Diagnostic Questionnaire 
(YDQ). Risk-behaviors were assessed using questions procured from the Global School-Based Student Health Survey (GSHS). A total of 11,931 adolescents were included in the analyses: $43.4 \%$ male and $56.6 \%$ female (M/F: 5179/6752), with a mean age of $14.89 \pm 0.87$ years. Adolescents reporting poor sleeping habits and risk-taking actions showed the strongest associations with PIU, followed by tobacco use, poor nutrition and physical inactivity. Among adolescents in the PIU group, 89.9\% were characterized as having multiple risk-behaviors. The significant association observed between PIU and risk-behaviors, combined with a high rate of co-occurrence, underlines the importance of considering PIU when screening, treating or preventing high-risk behaviors among adolescents.

Keywords: pathological Internet use; Internet addiction; risk-behavior; multiple risk-behaviors; unhealthy lifestyles; adolescents; SEYLE

\section{Introduction}

Adolescence is a transitional period characterized by considerable changes in physical, social and psychological attributes [1]. Moreover, relationships with peers, family and society undergo distinct changes during this transient period, as adolescents begin to assert autonomy over their decisions, emotions and behaviors [2]. Social aptitudes in adolescents often develop in the course of psychosocial interactions within different learning contexts [3]. Given the extensive platform for fostering social cognition and interpersonal skills [4,5], the Internet has proven to be a new and unique channel for psychosocial development among adolescents $[6,7]$.

Despite these inherent advantages, studies have shown that frequent and prolonged use of online applications has the propensity to displace conventional social interactions and relationships $[8,9]$. There is evidence demonstrating that accumulative time online displaces time on face-to-face interaction with family and friends [10], participating in extra-curricular activities [11], completing academic tasks [12], proper eating habits [13], physical activity [14] and sleeping [15]. As adolescents are spending more time online, there is a risk that their Internet use can become excessive or even pathological [16].

Pathological Internet use (PIU) is characterized by excessive or poorly-controlled preoccupations, urges or behaviors regarding Internet use that lead to impairment or distress [17]. PIU has conceptually been modelled as an impulse-control disorder and classified as a taxonomy of behavioral addiction akin to the nature of pathological gambling [18]. Despite recent advancements in PIU research, efforts to understand this phenomenon are impeded by the lack of international consensus on the diagnostic criteria of the condition. It is neither listed in the Diagnostic and Statistical Manual of Mental Disorders (DSM) nor the International Classification of Diseases (ICD) nosological systems. The major challenge facing PIU research is its conception as an addictive disorder.

In light of these contentions, the recently-published DSM-5 [19] has included behavioral addiction (non-substance-related addictive disorders) as an official diagnostic category, with gambling disorder (GD) being the only condition listed in this new classification. Internet gaming disorder (IGD) is also a potential subtype of behavioral addiction that was considered for inclusion in the DSM nosological system; however, evidence supporting IGD as a diagnostic disorder was still lacking. IGD was subsequently included into Section III of the DSM-5, as a condition that required further study [20], in order to determine its eventual suitability as a diagnostic disorder. In spite of the current nosological ambiguity of PIU, there continues to be surmounting evidence showing a strong link between PIU and other forms of addiction [21-24].

Research shows that individuals with PIU share neurological, biological and psychosocial attributes with both behavioral and substance-related addictions [25-29]. Based on a theoretical model denoted by Griffiths [30], there are six core symptoms exhibited in addictive disorders that are applicable to PIU. These include: salience (preoccupation with online activities), mood modification 
(using the Internet to escape or alleviate stress), tolerance (necessity to stay online longer), withdrawal (depression and irritability when offline), conflicts (interpersonal and intrapsychic) and relapse (failed attempts to discontinue Internet use). These core components provide a theoretical framework for estimating the magnitude of PIU.

Prevalence rates for PIU vary considerably across countries, partly due to the heterogeneity of its definition, nomenclature and diagnostic assessment. In an effort to estimate a global prevalence, Cheng and $\mathrm{Li}[31$ ] addressed these discrepancies by applying a random effects meta-analysis using studies with comparable psychometric instruments and criteria. This approach yielded a total of 89,281 participants from 31 countries spanning across several world regions. Results showed that the global prevalence of PIU was 6.0\% (95\% CI: 5.1-6.9) with only moderate heterogeneity.

Prevalence studies assessing PIU at the European level using representative samples are limited. Despite this paucity, there is emerging epidemiological evidence indicating stable trends in prevalence rates among this target group. In a representative sample of European adolescents $(n=18,709)$ aged 11-16 years, Blinka et al. [32] showed that the prevalence of PIU was $1.4 \%$. This coincides with rates reported by Tsitsika et al. [33], who estimated a PIU prevalence of $1.2 \%$ in a representative sample of European youth ( $n=13,284)$ aged 14-17 years. Durkee and colleagues [34], however, observed a slightly higher PIU prevalence of $4.4 \%$ in a representative sample of European adolescents $(n=11,956)$ aged 14-16 years. Prevalence rates for PIU in Europe were shown to be significantly higher in males than females, increase with age, differ by country and linked with an array of mental and behavioral disorders [35-39].

The onset of risk-behaviors frequently occurs during adolescence with a high likelihood of continuity into adulthood. Males tend to have a higher prevalence than females, and the frequency of risk-behaviors tends to increase with age [40]. There are distinct levels of severity ranging from low-risk (poor sleeping habits, poor nutrition and physical inactivity) to high-risk (excessive alcohol use, illicit drug use and tobacco use) behaviors. Research has typically assessed risk-behaviors as independent entities, albeit that clear evidence shows their co-occurrence, even at an early age [41,42]. Populations with multiple risk-behaviors have the greatest risk for chronic diseases, psychiatric disorders, suicidal behaviors and premature death compared to individuals with single or no risk-behaviors $[43,44]$. Given the concurrent nature of risk-behaviors, it is imperative to understand their implication on adolescents' risk of PIU.

The Youth Risk Behavior Surveillance System (YRBSS) in the U.S. ascertains that risk-behaviors are a major contributor to the leading causes of morbidity among adolescents and young people [45]. Apart from this implicit supposition, there is relatively little research that systematically scrutinizes the extent to which these forms of behavior relate to adolescent PIU, particularly within the European context. Epidemiological investigations are necessary in order to acquire a better understanding of this phenomenon.

Based on a large, representative sample of school-based adolescents in Europe, the primary objective of this study is to investigate the association between risk-behaviors (i.e., alcohol use, illicit drug use, tobacco use, risk-taking actions, truancy, poor sleeping habits, poor nutrition and physical inactivity) and distinct forms of Internet use.

\section{Materials and Methods}

\subsection{Study Design and Population}

The present cross-sectional study was performed within the framework of the European Union project: Saving and Empowering Young Lives in Europe (SEYLE) [46]. Adolescents were recruited from randomly-selected schools across study sites in Austria, Estonia, France, Germany, Hungary, Ireland, Israel, Italy, Romania, Slovenia and Spain, with Sweden serving as the coordinating center.

The inclusion criteria for selecting eligible schools were based on the following conditions: (1) schools were public; (2) contained at least 40 students aged 15 years; (3) had more than two teachers 
for students aged 15 years; and (4) had no more than $60 \%$ of students of the same gender. Eligible schools were categorized by size: (i) small ( $\leqslant$ the median number of students in all schools of the study site); and (ii) large ( $\geqslant$ the median number of students in all schools of the study site) [46]. Using a random number generator, schools were randomized according to SEYLE interventions and school size with respect to sociocultural factors, school environment and school system structure in each study site.

Data were collected through structured questionnaires administered to adolescents within the school milieu. Representativeness, consent, participation and response rates of the sample are reported in a methodological analysis [47].

The present study was conducted in accordance with the Declaration of Helsinki, and the protocol was approved by the local Ethics Committee in each participating country (Project No. HEALTH-F2-2009-223091). Prior to participating in the study, both adolescents and parents provided their informed consent for participation.

\subsection{Measurements}

PIU was assessed using Young's Diagnostic Questionnaire (YDQ) [18]. The YDQ is an 8-item questionnaire assessing patterns of Internet usage that result in psychological or social impairment during the six-month period preceding data collection [48]. The eight items in the YDQ correspond to the six items in Griffiths' components model and nine items in the diagnostic criteria of IGD in the DSM-5 [49,50]. Based on the YDQ score, ranging from 0-8, Internet users were categorized into three groups: adaptive Internet users (AIU) (scoring 0-2); maladaptive Internet users (MIU) (scoring 3-4); and pathological Internet users (PIU) (scoring $\geqslant 5$ ) [51]. Moreover, hours online per day were measured using a single-item question in the structured questionnaire.

Data on risk-behaviors were obtained by using questions from the Global School-Based Student Health Survey (GSHS) [52]. Developed by the World Health Organization (WHO) and collaborators, the GSHS is a school-based survey assessing health risk-behaviors among adolescents aged 13-17 years. This self-report questionnaire comprises items that correspond to the 10 leading causes of morbidity among adolescents and young people.

\subsection{Individual Risk-Behaviors}

Based on the GSHS, individual risk-behaviors were delineated into three categories: (i) substance use; (ii) sensation-seeking; (iii) and lifestyle characteristics. The ensuing individual risk-behaviors were coded as dichotomous variables.

\subsubsection{Substance Use}

Substance use involved alcohol use, illicit drug use and use of tobacco. The variables were classified accordingly: (1) frequency of alcohol use: $\geqslant 2$ times/week $v s . \leqslant 1$ times/week; (2) number of drinks on a typical drinking day: $\geqslant 3$ drinks $v s . \leqslant 2$ drinks; (3) lifetime incidence of drinking to the point of drunkenness (alcohol intoxication): $\geqslant 3$ times $v s . \leqslant 2$ times; (4) lifetime incidence of having a hangover after drinking: $\geqslant 3$ times $v s . \leqslant 2$ times; (5) ever used drugs: yes/no; (6) ever used hashish or marijuana: yes/no; (7) ever used tobacco: yes/no; and (8) currently smoking cigarettes: $\geqslant 6 /$ day vs. $\leqslant 5 /$ day.

\subsubsection{Sensation-Seeking}

Sensation-seeking comprised four items indicating risk-taking actions during the past twelve months: (1) driven in a vehicle by a friend who had been drinking alcohol; (2) ridden a skateboard or roller-bladed in traffic without a helmet and/or (3) pulled along a moving vehicle; and (4) gone to dangerous streets or alleys during night time. Response alternatives were yes/no in all four items. 


\subsubsection{Lifestyle Characteristics}

Lifestyle characteristics included variables related to sleep, nutrition, physical activity and school attendance. Sleeping habits referred to the past six months: (1) feeling tired in the morning before school: $\geqslant 3$ days/week $v$ s. $\leqslant 2$ days/week; (2) napping after school: $\geqslant 3$ days/week $v$ s. $\leqslant 2$ days/week; and (4) sleeping: $\leqslant 6$ hours/night $v s$. $\geqslant 7$ hours/night. Nutrition referred to the past six months: (4) consuming fruits/vegetables: $\leqslant 1$ time/week vs. $\geqslant 2$ times/week; and (5) consuming breakfast before school: $\leqslant 2$ days/week vs. $\geqslant 3$ days/week. Physical activity referred to the past six months: (6) physical activity for at least 60 minutes during the past two weeks: $\leqslant 3$ days $v s$. $\geqslant 4$ days; and (7) playing sports on a regular basis: yes/no. School attendance comprised one item on the occurrence of unexcused absences from school during the past two weeks: $\geqslant 3$ days vs. $\leqslant 2$ days.

\subsection{Multiple Risk-Behaviors}

The total number of risk-behaviors was calculated into a single variable and coded as an ordinal measure. Split-half reliability $\left(r_{s b}=0.742\right)$ and internal consistency $(\alpha=0.714)$ values indicated an acceptable level of homogeneity between items in the multiple risk-behavior measure.

\section{Statistical Analyses}

The prevalence of individual risk-behaviors among Internet user groups was calculated for males and females. To ascertain statistically-significant differences between group proportions, multiple pairwise comparisons using the two-sided z-test with Bonferroni adjusted $p$-values was performed. Extended analyses were conducted to test the effect of individual risk-behaviors on MIU and PIU using generalized linear mixed models (GLMM) with a multinomial logit link and full maximum likelihood estimation. In the GLMM analysis, MIU and PIU were entered as the outcome measures with AIU as the reference category, individual risk-behaviors were entered as Level 1 fixed effects, school as Level 2 random intercept and country as Level 3 random intercept. Variance components were used as the covariance structure for the random effects. To study the moderating effect of gender, interaction terms (gender * risk-behavior) were fitted into the regression model. Adjustments for age and gender were applied to relevant GLMM models. Odds ratios $(O R)$ with $95 \%$ confidence intervals $(C I)$ are reported for the respective models.

In the analysis on multiple risk-behaviors, the mean $(M)$ and standard error of the mean (SEM) were calculated for the different Internet user groups and stratified by gender. Box and whisker plots were used to illustrate these relationships. Statistical significance between multiple risk-behaviors and gender was assessed using independent samples $t$-test. One-way analysis of variance (ANOVA) with post hoc pairwise comparisons was employed to assess the statistical significance between multiple risk-behaviors and Internet user groups.

A regression variable plot was conducted to elucidate the linear relationship between the number of hours online per day and the number of risk-behaviors among Internet user groups. All statistical tests were performed using IBM SPSS Statistics 23.0. A critical value of $p<0.05$ was considered to be statistically significant.

\section{Results}

\subsection{Characteristics of the Study Sample}

Among the initial SEYLE sample of 12,395 adolescents, there were 464 (3.7\%) subjects excluded due to missing data on relevant variables. This yielded a sample size of 11,931 school-based adolescents for the present study. The sample comprised $43.4 \%$ male and 56.6\% female adolescents (M/F: 5179/6752) with a mean age of $14.89 \pm 0.87$ years. The prevalence of MIU was significantly higher among females $(14.3 \%)$ compared to males (12.4\%), whereas PIU was significantly higher among males $(5.2 \%)$ than females $(3.9 \%)\left(\chi^{2}(2,11928)=19.92, p<0.001\right)$. 


\subsection{Prevalence of Risk-Behaviors}

Table 1 describes the prevalence of risk-behaviors stratified by Internet user group. The average prevalence rates among Internet user groups (AIU, MIU and PIU) were $16.4 \%, 24.3 \%$ and $26.5 \%$ for substance use (alcohol use, illicit drug use and tobacco use); $19.0 \%, 27.8 \%$ and $33.8 \%$ for sensation-seeking behaviors (risk-taking actions); and $23.8 \%, 30.8 \%$ and $35.2 \%$ for lifestyle characteristics (poor sleeping habits, poor nutrition, physical inactivity and truancy), respectively. Prevalence within MIU and PIU groups was significantly higher compared to the AIU group in all risk categories (substance use, sensation-seeking and lifestyle characteristics). With the exception of five subcategories, pairwise comparisons showed that prevalence rates did not significantly differ between MIU and PIU groups.

\subsection{Multiple Risk-Behaviors}

Results showed that $89.9 \%$ of adolescents in the PIU group reported multiple risk-behaviors. The one-way ANOVA test revealed that the mean rate of multiple risk-behaviors significantly increased from adaptive use $(\mathrm{M}=4.89, \mathrm{SEM}=0.02)$ to maladaptive use $(\mathrm{M}=6.38, \mathrm{SEM}=0.07)$ to pathological use $(\mathrm{M}=7.09, \mathrm{SEM}=0.12)(\mathrm{F}(2,11928)=310.35, p<0.001)$. This trend was virtually equivalent for males and females (Figure 1).

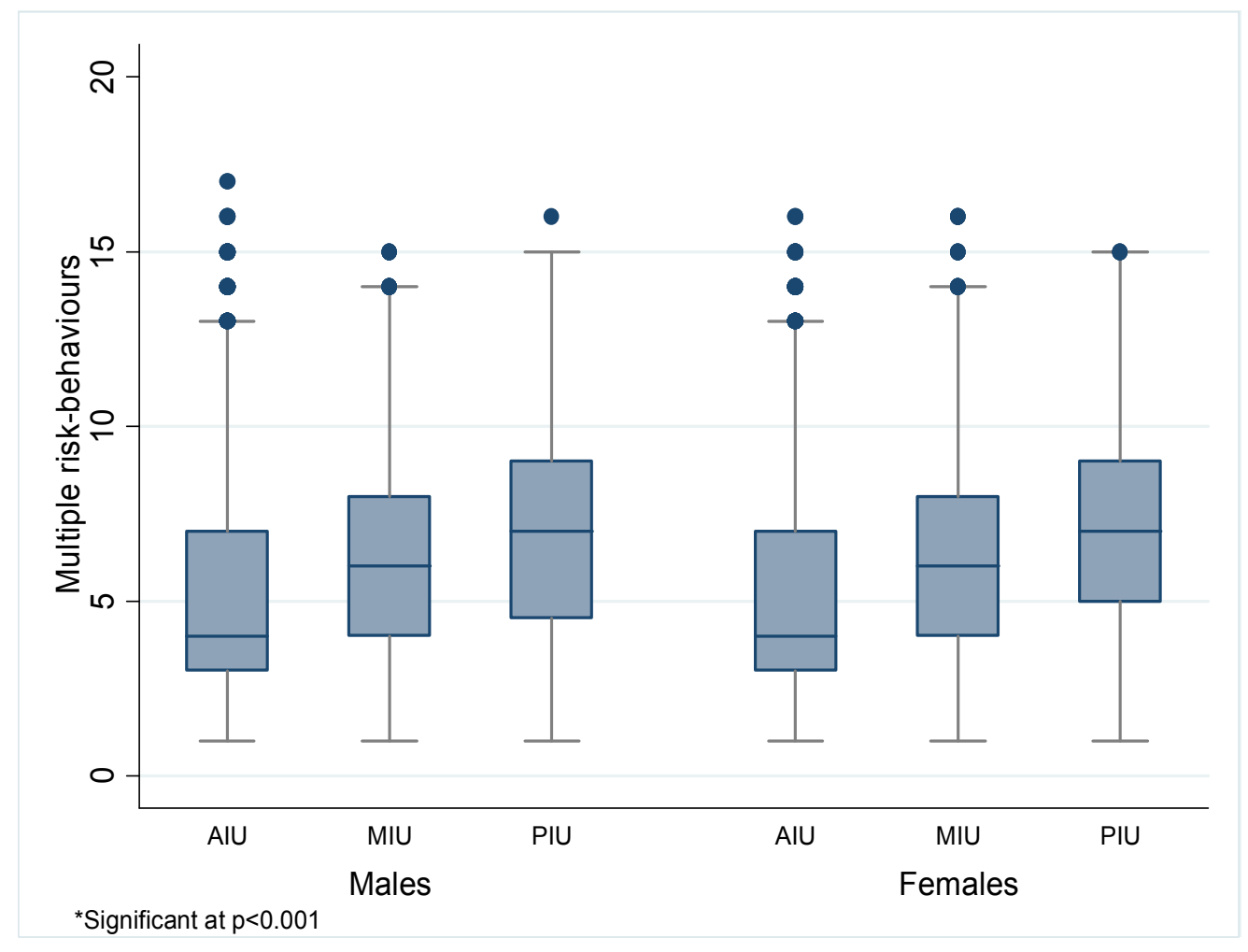

Figure 1. Box and whisker plot of multiple risk-behaviors among adaptive Internet users (AIU), maladaptive Internet users (MIU) and pathological Internet users (PIU) stratified by gender *

Moreover, no statistical difference between genders in both MIU $(t(1608)=0.529, p=0.597)$ and PIU $(t(526)=1.92, p=0.054)$ groups were observed (Table 2). It should be noted, however, that the $p$-value for the PIU group was relatively close to reaching statistical significance $(p=0.054)$.

The regression variable plot exhibited a clear linear relationship between the number of hours online per day and the number of risk-behaviors in adolescents. This trend was comparatively identical between Internet user groups (Figure 2). 
Table 1. Prevalence of risk-behaviors among adolescents stratified by gender and Internet user group $1,2 a-c$.

\begin{tabular}{|c|c|c|c|c|c|c|c|c|c|c|c|}
\hline \multicolumn{3}{|c|}{ Prevalence of Risk-Behaviors among Adolescents } & \multicolumn{3}{|c|}{ Adaptive Use } & \multicolumn{3}{|c|}{ Maladaptive Use } & \multicolumn{3}{|c|}{ Pathological Use } \\
\hline & & & Male & Female & Total & Male & Female & Total & Male & Female & Total \\
\hline RCAT & Risk-Behaviors & Subcategories & $\%$ & $\%$ & $\%$ & $\%$ & $\%$ & $\%$ & $\%$ & $\%$ & $\%$ \\
\hline \multirow{8}{*}{ 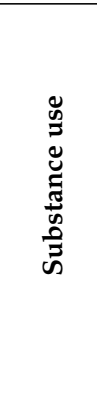 } & \multirow{4}{*}{ Alcohol Use } & Drinking alcohol $\geqslant 2$ times/week & $10.6^{\mathrm{a}}$ & $5.7^{\mathrm{b}}$ & $7.9^{\mathrm{a}}$ & $15.6^{\mathrm{a}}$ & $9.4^{\mathrm{b}}$ & $11.9^{\mathrm{b}}$ & $16.4^{\mathrm{a}}$ & $11.2^{\mathrm{a}}$ & $13.8^{\mathrm{b}}$ \\
\hline & & $\geqslant 3$ drinks on a typical drinking day & $24.0^{\mathrm{a}}$ & $20.0^{\mathrm{b}}$ & $21.8^{\mathrm{a}}$ & $31.3^{\mathrm{a}}$ & $31.4^{\mathrm{a}}$ & $31.4^{\mathrm{b}}$ & $34.3^{\mathrm{a}}$ & $38.1^{\mathrm{a}}$ & $36.2^{b}$ \\
\hline & & Alcohol intoxication $\geqslant 3$ times/lifetime & $16.1^{\mathrm{a}}$ & $10.5^{\mathrm{b}}$ & $13.0^{\mathrm{a}}$ & $25.1^{\mathrm{a}}$ & $18.1^{\mathrm{b}}$ & $20.9^{b}$ & $25.4^{\mathrm{a}}$ & $25.0^{\mathrm{a}}$ & $25.2^{b}$ \\
\hline & & Hangover after drinking $\geqslant 3$ times/lifetime & $8.5^{\mathrm{a}}$ & $6.0^{\mathrm{b}}$ & $7.1^{\mathrm{a}}$ & $15.6^{\mathrm{a}}$ & $9.4^{\mathrm{b}}$ & $11.9^{\mathrm{b}}$ & $13.8^{\mathrm{a}}$ & $15.4^{\mathrm{a}}$ & $14.6^{b}$ \\
\hline & \multirow{2}{*}{ Illicit Drug Use } & Have used drugs during lifetime & $6.1^{\mathrm{a}}$ & $3.7^{\mathrm{b}}$ & $4.8^{\mathrm{a}}$ & $11.1^{\mathrm{a}}$ & $5.7^{\mathrm{b}}$ & $7.8^{\mathrm{b}}$ & $7.5^{\mathrm{a}}$ & $8.8^{\mathrm{a}}$ & $8.1^{\mathrm{b}}$ \\
\hline & & Have used hashish or marijuana during lifetime & $10.6^{\mathrm{a}}$ & $6.7^{b}$ & $8.4^{\mathrm{a}}$ & $15.4^{\mathrm{a}}$ & $10.5^{b}$ & $12.5^{\mathrm{b}}$ & $15.3^{\mathrm{a}}$ & $15.2^{\mathrm{a}}$ & $15.3^{\mathrm{b}}$ \\
\hline & \multirow{2}{*}{ Tobacco Use } & Have smoked cigarettes during lifetime & $41.2^{\mathrm{a}}$ & $44.0^{\mathrm{b}}$ & $42.8^{\mathrm{a}}$ & $55.8^{\mathrm{a}}$ & $61.4^{\mathrm{b}}$ & $59.1^{b}$ & $60.1^{\mathrm{a}}$ & $65.8^{\mathrm{a}}$ & $62.9^{b}$ \\
\hline & & Currently smoking $\geqslant 6$ cigarettes/day & $26.1^{\mathrm{a}}$ & $31.2^{b}$ & $29.0^{\mathrm{a}}$ & $31.6^{\mathrm{a}}$ & $43.5^{b}$ & $38.8^{\mathrm{b}}$ & $31.3^{\mathrm{a}}$ & $41.2^{b}$ & $36.2^{b}$ \\
\hline \multirow{3}{*}{ 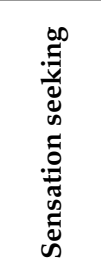 } & \multirow{3}{*}{$\begin{array}{l}\text { Risk-Taking } \\
\text { Actions }\end{array}$} & $\begin{array}{l}\text { Driven in a vehicle by a friend who has been drinking alcohol } \\
\text { during lifetime }\end{array}$ & $16.5^{\mathrm{a}}$ & $14.1^{\mathrm{b}}$ & $15.2^{\mathrm{a}}$ & $24.1^{\mathrm{a}}$ & $20.8^{\mathrm{a}}$ & $22.1^{\mathrm{b}}$ & $28.7^{\mathrm{a}}$ & $29.6^{\mathrm{a}}$ & $29.2^{c}$ \\
\hline & & $\begin{array}{l}\text { Ridden skateboard or roller-blades in traffic without a helmet } \\
\text { during lifetime }\end{array}$ & $31.1^{\mathrm{a}}$ & $24.2^{b}$ & $27.2^{\mathrm{a}}$ & $37.7^{\mathrm{a}}$ & $36.1^{\mathrm{a}}$ & $36.7^{\mathrm{b}}$ & $35.4^{\mathrm{a}}$ & $43.5^{\mathrm{a}}$ & $39.4^{b}$ \\
\hline & & Pulled along a moving vehicle during lifetime & $7.8^{\mathrm{a}}$ & $2.3^{b}$ & $4.7^{\mathrm{a}}$ & $12.8^{a}$ & $4.9^{\mathrm{b}}$ & $8.0^{\mathrm{b}}$ & $19.8^{\mathrm{a}}$ & $6.5^{b}$ & $13.3^{c}$ \\
\hline \multirow{8}{*}{ 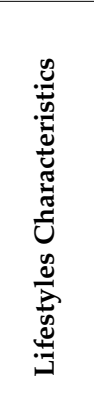 } & Truancy & Unexcused absences from school $\geqslant 3$ days/two-weeks & $3.9^{\mathrm{a}}$ & $2.2^{b}$ & $3.0^{\mathrm{a}}$ & $9.0^{\mathrm{a}}$ & $4.6^{\mathrm{b}}$ & $6.4^{\mathrm{b}}$ & $11.9^{\mathrm{a}}$ & $5.8^{\mathrm{b}}$ & $8.9^{b}$ \\
\hline & \multirow{3}{*}{$\begin{array}{l}\text { Poor Sleeping } \\
\text { Habits }\end{array}$} & Feeling tired in the morning before school $\geqslant 3$ days/week & $52.7^{\mathrm{a}}$ & $57.4^{\mathrm{b}}$ & $55.4^{\mathrm{a}}$ & $70.1^{\mathrm{a}}$ & $74.4^{\mathrm{a}}$ & $72.7^{b}$ & $71.6^{\mathrm{a}}$ & $82.7^{b}$ & $77.1^{\mathrm{b}}$ \\
\hline & & Napping after school $\geqslant 3$ days/week & $21.1^{\mathrm{a}}$ & $19.0^{\mathrm{a}}$ & $19.8^{\mathrm{a}}$ & $26.6^{\mathrm{a}}$ & $24.1^{\mathrm{a}}$ & $25.1^{\mathrm{b}}$ & $36.5^{\mathrm{a}}$ & $23.7^{b}$ & $30.7^{b}$ \\
\hline & & Sleeping $\leqslant 6 \mathrm{~h} /$ night & $11.8^{\mathrm{a}}$ & $15.0^{\mathrm{b}}$ & $13.6^{\mathrm{a}}$ & $16.7^{\mathrm{a}}$ & $26.3^{b}$ & $22.5^{\mathrm{b}}$ & $23.9^{\mathrm{a}}$ & $35.8^{\mathrm{b}}$ & $29.7^{c}$ \\
\hline & \multirow{2}{*}{ Poor Nutrition } & Consuming fruits and vegetables $\leqslant 1$ time/week & $16.9^{\mathrm{a}}$ & $11.6^{\mathrm{b}}$ & $13.9^{\mathrm{a}}$ & $24.8^{\mathrm{a}}$ & $18.1^{\mathrm{b}}$ & $20.7^{b}$ & $32.5^{\mathrm{a}}$ & $20.0^{b}$ & $26.3^{c}$ \\
\hline & & Consuming breakfast before school $\leqslant 2$ days/week & $31.0^{\mathrm{a}}$ & $41.1^{\mathrm{b}}$ & $36.7^{\mathrm{a}}$ & $36.1^{\mathrm{a}}$ & $49.1^{b}$ & $43.9^{b}$ & $41.0^{\mathrm{a}}$ & $56.5^{b}$ & $48.7^{b}$ \\
\hline & \multirow{2}{*}{ Physical Inactive } & Physical activity $\leqslant 3$ days/two-weeks & $13.2^{\mathrm{a}}$ & $22.4^{b}$ & $18.4^{\mathrm{a}}$ & $18.5^{\mathrm{a}}$ & $23.7^{b}$ & $21.6^{\mathrm{b}}$ & $20.9^{\mathrm{a}}$ & $26.5^{\mathrm{a}}$ & $23.7^{b}$ \\
\hline & & Does not play sport(s) on a regular basis & $20.2^{\mathrm{a}}$ & $37.2^{b}$ & $29.8^{\mathrm{a}}$ & $24.8^{\mathrm{a}}$ & $39.2^{b}$ & $33.4^{\mathrm{b}}$ & $28.0^{\mathrm{a}}$ & $44.6^{b}$ & $36.2^{b}$ \\
\hline
\end{tabular}

${ }^{1} \mathrm{~N}=11,931$ (AIU = 9793 (M/F: 4269/5524), MIU = 1610 (M/F: 642/968), PIU = 528 (M/F: 268/260)); RCAT = risk categories; ${ }^{2 a}$ Gender values (a and b) in the same row and sub-table not sharing the same subscript indicate significant differences at $p<0.05 .{ }^{2 b}$ Total column values $(\mathrm{a}, \mathrm{b}, \mathrm{c})$ in the same row not sharing the same subscript indicate significant differences between Internet user groups at $p<0.05 .{ }^{2 \mathrm{c}}$ Multiple pairwise comparisons were assessed using the two-sided z-test of proportions with Bonferroni-corrected $p$-values. 
Table 2. Independent samples $t$-test of multiple risk-behaviors and gender by Internet user group ${ }^{1-3}$.

\begin{tabular}{|c|c|c|c|c|c|c|c|c|c|c|}
\hline \multirow{3}{*}{$\begin{array}{l}\text { Internet User } \\
\text { Groups }\end{array}$} & \multicolumn{10}{|c|}{ Multiple Risk-Behaviors } \\
\hline & \multicolumn{10}{|c|}{$\begin{array}{l}\text { Homogeneity of } \\
\text { Variances }\end{array}$} \\
\hline & $\mathbf{F}$ & $p$-value & Gender & Mean & SEM & $t$ & df & $p$-value & $\begin{array}{c}\text { Mean } \\
\text { Difference }\end{array}$ & $\begin{array}{c}\text { SE } \\
\text { Difference }\end{array}$ \\
\hline \multirow{2}{*}{ Adaptive use } & \multirow{2}{*}{48.254} & \multirow{2}{*}{$<0.001$} & Male & 4.87 & 0.04 & \multirow{2}{*}{0.943} & \multirow{2}{*}{9791} & \multirow{2}{*}{0.345} & \multirow{2}{*}{0.055} & \multirow{2}{*}{0.058} \\
\hline & & & Female & 4.92 & 0.03 & & & & & \\
\hline \multirow{2}{*}{ Maladaptive use } & \multirow{2}{*}{2.238} & \multirow{2}{*}{0.135} & Male & 6.33 & 0.11 & \multirow{2}{*}{0.529} & \multirow{2}{*}{1608} & \multirow{2}{*}{0.597} & \multirow{2}{*}{0.077} & \multirow{2}{*}{0.146} \\
\hline & & & Female & 6.41 & 0.09 & & & & & \\
\hline \multirow{2}{*}{ Pathological use } & \multirow{2}{*}{0.060} & \multirow{2}{*}{0.806} & Male & 6.85 & 0.18 & \multirow{2}{*}{1.928} & \multirow{2}{*}{526} & \multirow{2}{*}{0.054} & \multirow{2}{*}{0.492} & \multirow{2}{*}{0.255} \\
\hline & & & Female & 7.34 & 0.18 & & & & & \\
\hline
\end{tabular}

${ }^{1} \mathrm{~N}=11,931$ (AIU = 9793 (M/F: 4269/5524), MIU = 1610 (M/F: 642/968), PIU = 528 (M/F: 268/260). ${ }^{2}$ Model abbreviations include the F-statistic (F), standard error of the mean (SEM), standard error (SE), $t$-statistic $(t)$ and degrees of freedom (df). ${ }^{3}$ Levene's test for equality of variances was performed to assess homogeneity $(p>0.05$ indicates equal variances).

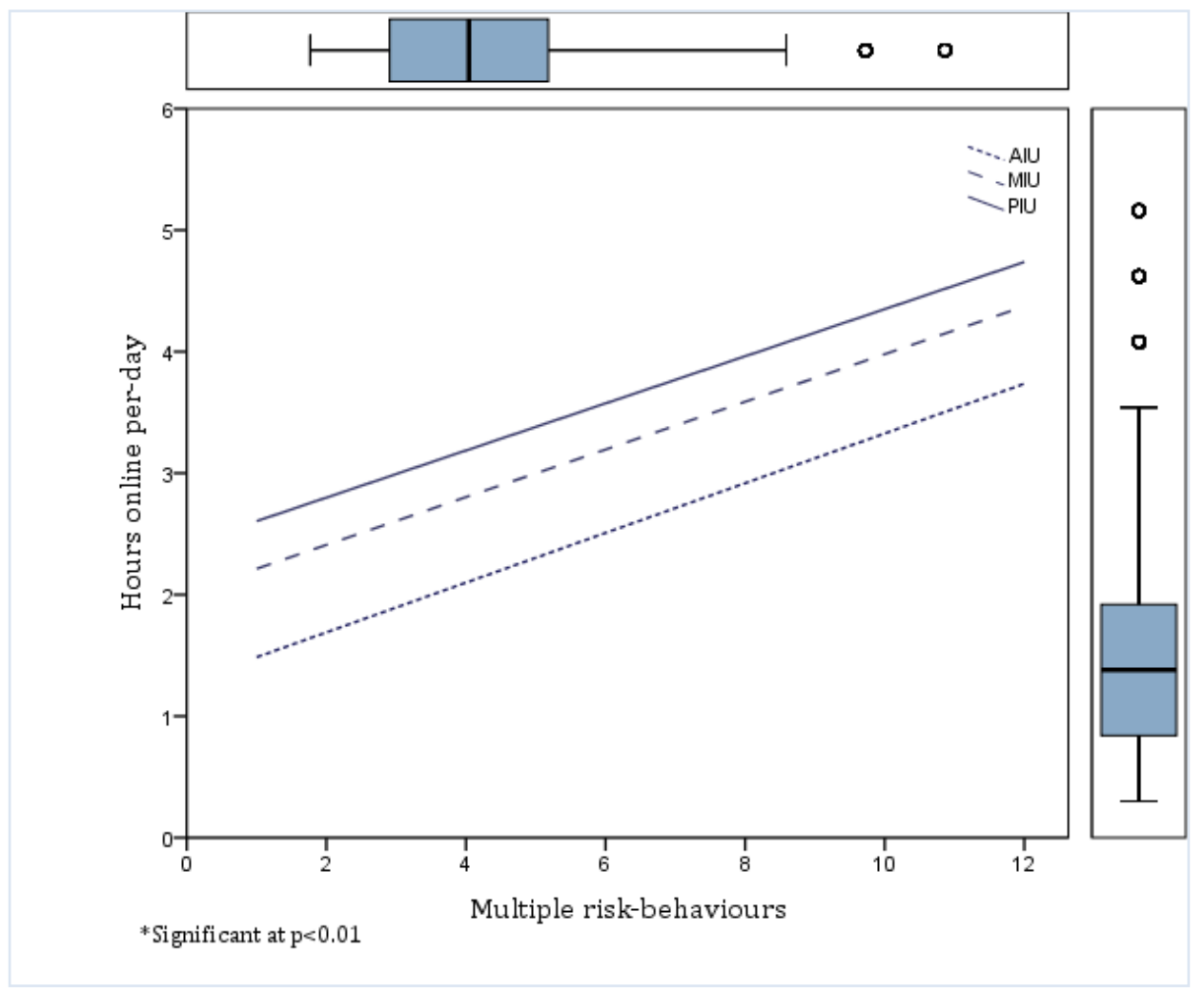

Figure 2. Linear relationship between the number of hours online per day and the number of risk-behaviors among AIU, MIU and PIU groups *.

\subsection{GLMM Analysis of the Association between Risk-Behaviors, MIU and PIU}

Risk-behaviors that were significantly associated with MIU were also significantly associated with PIU, with the exception of three subcategories noted within risk-taking actions and truancy (Table 3). The GLMM analysis showed that all subcategories of poor sleeping habits significantly increased the relative odds of PIU with effect sizes ranging from $O R=1.45$ to $O R=2.17$. Significant associations were observed between risk-taking actions and PIU with effect sizes ranging from $O R=1.55$ to $O R=1.73$. Moreover, odds ratios for single subcategories within the tobacco use $(O R=1.41)$, poor nutrition $(O R=1.41)$ and physical inactivity $(O R=1.39)$ domains were statistically significant. 
Table 3. Generalized linear mixed model (GLMM) of the association between individual risk-behaviors, maladaptive use and pathological use with an extended analysis on gender interactions ${ }^{1-4}$.

\begin{tabular}{|c|c|c|c|c|c|c|c|c|c|}
\hline \multirow{2}{*}{ RCAT $^{a}$} & \multirow{2}{*}{ Risk-Behaviors } & \multirow{2}{*}{ Subcategories } & \multicolumn{3}{|c|}{ Maladaptive Use ${ }^{\mathbf{b}}$} & \multicolumn{3}{|c|}{ Pathological Use $^{\mathrm{c}}$} & \multirow{2}{*}{$\begin{array}{c}\text { Gender } \\
\text { Interactions }^{\mathrm{d}}\end{array}$} \\
\hline & & & OR & $95 \%$ CI & $p$-Value & OR & $95 \%$ CI & $p$-Value & \\
\hline \multirow{8}{*}{ 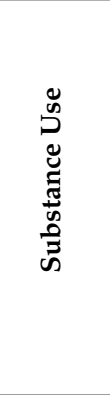 } & \multirow{4}{*}{ Alcohol Use } & Drinking alcohol $\geqslant 2$ times/week & 1.00 & $0.82-1.22$ & 0.943 & 0.88 & $0.64-1.20$ & 0.426 & n.s. \\
\hline & & $\geqslant 3$ drinks on a typical drinking day & 1.07 & $0.92-1.24$ & 0.336 & 1.25 & $0.99-1.59$ & 0.060 & n.s. \\
\hline & & Alcohol intoxication $\geqslant 3$ times / lifetime & 1.03 & $0.85-1.24$ & 0.718 & 1.07 & $0.80-1.43$ & 0.646 & n.s. \\
\hline & & Hangover after drinking $\geqslant 3$ times/lifetime & 1.01 & $0.82-1.26$ & 0.872 & 0.98 & $0.71-1.36$ & 0.920 & n.s. \\
\hline & \multirow{2}{*}{ Illicit Drug Use } & Have used drugs during lifetime & 1.07 & $0.82-1.41$ & 0.583 & 0.81 & $0.53-1.22$ & 0.322 & n.s. \\
\hline & & Have used hashish or marijuana during lifetime & 0.85 & $0.68-1.07$ & 0.178 & 0.93 & $0.67-1.30$ & 0.695 & n.s. \\
\hline & \multirow{2}{*}{ Tobacco Use } & Have smoked cigarettes during lifetime & 1.29 & $1.08-1.55$ & 0.004 & 1.41 & $1.06-1.88$ & 0.018 & Male* \\
\hline & & Currently smoking $\geqslant 6$ cigarettes/day & 1.06 & $0.90-1.25$ & 0.456 & 0.92 & $0.71-1.20$ & 0.575 & n.s. \\
\hline \multirow{4}{*}{ 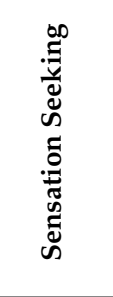 } & \multirow{4}{*}{$\begin{array}{l}\text { Risk-Taking } \\
\text { Actions }\end{array}$} & $\begin{array}{l}\text { Driven in a vehicle by a friend who has been } \\
\text { drinking alcohol during lifetime }\end{array}$ & 1.14 & $0.99-1.32$ & 0.060 & 1.55 & $1.24-1.94$ & $<0.001$ & Female * \\
\hline & & $\begin{array}{l}\text { Ridden skateboard or roller-blades in traffic } \\
\text { without a helmet during lifetime }\end{array}$ & 1.18 & $1.04-1.34$ & 0.008 & 1.07 & $0.87-1.32$ & 0.480 & Female ** \\
\hline & & Pulled along a moving vehicle during lifetime & 1.24 & $0.99-1.55$ & 0.055 & 1.64 & $1.20-2.24$ & 0.002 & Male *** \\
\hline & & $\begin{array}{l}\text { Gone to dangerous streets or alleys at night-time } \\
\text { during lifetime }\end{array}$ & 1.46 & $1.29-1.66$ & $<0.001$ & 1.73 & $1.41-2.14$ & $<0.001$ & Female $* * *$ \\
\hline \multirow{8}{*}{ 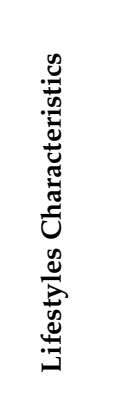 } & Truancy & Unexcused absences $\geqslant 3$ days/two-weeks & 1.39 & $1.08-1.79$ & 0.010 & 1.22 & $0.85-1.75$ & 0.268 & Male * \\
\hline & \multirow{3}{*}{$\begin{array}{l}\text { Poor Sleeping } \\
\text { Habits }\end{array}$} & $\begin{array}{l}\text { Feeling tired in the morning before school } \\
\geqslant 3 \text { days/week }\end{array}$ & 1.77 & $1.57-2.01$ & $<0.001$ & 2.17 & $1.74-2.72$ & $<0.001$ & Female *** \\
\hline & & Napping after school $\geqslant 3$ days/week & 1.27 & $1.12-1.36$ & 0.024 & 1.45 & $1.19-1.77$ & $<0.001$ & Male $* * *$ \\
\hline & & Sleeping $\leqslant 6$ hrs $/$ night & 1.29 & $1.12-1.48$ & $<0.001$ & 1.80 & $1.45-2.24$ & $<0.001$ & Female *** \\
\hline & \multirow{2}{*}{ Poor Nutrition } & Consuming fruits and vegetables $\leqslant 1$ time/week & 1.34 & $1.16-1.55$ & $<0.001$ & 1.41 & $1.13-1.76$ & 0.002 & Male *** \\
\hline & & Consuming breakfast before school $\leqslant 2$ days/week & 1.03 & $0.92-1.16$ & 0.566 & 1.17 & $0.96-1.42$ & 0.114 & n.s. \\
\hline & \multirow{2}{*}{ Physical Inactivity } & Physical activity $\leqslant 3$ days/two-weeks & 1.18 & $1.02-1.36$ & 0.024 & 1.39 & $1.10-1.76$ & 0.006 & n.s. \\
\hline & & Does not play sport(s) on a regular basis & 1.02 & $0.90-1.16$ & 0.715 & 1.12 & $0.91-1.38$ & 0.264 & n.s. \\
\hline
\end{tabular}

${ }^{1} \mathrm{~N}=11,931$ (AIU $=9793, \mathrm{MIU}=1610$, PIU $\left.=528\right) .{ }^{2}$ Adaptive use is the reference category. ${ }^{3}$ Outcomes are presented as an odds ratio $(\mathrm{OR})$ with $95 \%$ confidence intervals $(95 \%$ CI) and $p$-values. ${ }^{4}$ Models are adjusted for age and gender. ${ }^{a}$ RCAT $=$ risk categories. ${ }^{b}$ Random-effects parameter (country ${ }^{*}$ school) $=0.133 ;$ CI: $0.008-0.203, p<0.001$. ${ }^{\mathrm{C}}$ Random-effects parameter (country ${ }^{*}$ school) $=0.356$; CI: $0.230-0.550, p<0.001 .{ }^{\mathrm{d}}$ Interaction terms (gender ${ }^{*}$ risk-behavior). n.s. $=$ not significant; ${ }^{*} p<0.05 ;{ }^{* *} p<0.01 ; * * * p<0.001$. 


\subsection{Gender Interactions}

The analysis on gender interactions revealed that the association between risk-taking actions, poor sleeping habits and PIU was significantly higher in females, whereas the association between truancy, poor nutrition and PIU was significantly higher in males (Table 3).

\section{Discussion}

\subsection{Prevalence of Risk-Behaviors}

The present study sought to examine the relationship between PIU and risk-behaviors. Results showed that the prevalence of risk-behaviors was significantly higher among pathological users compared to adaptive users with some variations between genders. The highest prevalence observed among maladaptive and pathological users was poor sleeping habits followed by tobacco use. These estimations are considerably higher compared to prevalence rates reported in studies conducted outside the EU, namely in the Asia and Pacific regions [53,54]. One plausible explanation could be related to the variations observed at the ecological level (e.g., penetration rates) among these respective regions. Statistics show that the European region has the highest Internet penetration rate $(78 \%)$ worldwide. European rates are more than double compared to those depicted in the Asia and Pacific regions (36\%) [55]. The actual role penetration rates have on influencing the prevalence of PIU remains ambiguous; thus, future efforts examining this relationship would be of great value for explaining this connection.

\subsection{Substance Use}

The characteristics between risk-behaviors and addictive behaviors are highly overlapping. This is perhaps most evident with substance use. Substance use is often classified as a risk-behavior; however, it is also an antecedent of substance abuse. If high-risk behaviors share similar underlying mechanisms, then having one problem behavior may lower the threshold to developing other problem behaviors. This assertion is corroborated by evidence-based research demonstrating a high level of interconnectedness between various risk-behaviors [56]. Based on this concept, it is plausible to assume that adolescents with pre-existing risk-behaviors are likely to have a higher risk of PIU compared to adolescents without risk-behaviors.

\subsection{Sensation-Seeking}

In line with the foregoing research [57], results showed that the majority of risk-taking actions within the sensation-seeking category were significantly associated with PIU. Sensation-seeking is a personality trait associated with deficiencies in self-regulation and deferred gratification [58]. These attributes among youth are often related to a perceptual predisposition of an 'optimistic bias effect' in which adolescents are more likely to discount risks for themselves, while overestimating risks for others [59]. Adolescents exhibiting these deflecting traits are likely to have a higher propensity for behavioral problems.

\subsection{Lifestyle Characteristics}

Poor sleeping habits proved to be the strongest factors related to PIU. This is likely due to a displacement effect of sleep for online activities. There are certain online activities that explicitly induce users to stay online longer than anticipated. A study on massively multiplayer online role-playing games (MMORPG) indicated that users are enticed to stay online longer in order to follow the progressive storyline of their online character [60]. Excessive use of social networking sites has also emerged in recent years, denoting both an increase in time spent online and negative correlations with real-life social interactions [61,62]. Studies show that adolescents excessively using the Internet have the propensity for developing sleeping disorders as a result of their extended time online $[63,64]$. 
The chronic displacement of sleep for online activities could lead to sleep deprivation, which is known to cause severe adverse effects on social, psychological and somatic functioning.

Disturbances in regulated sleep patterns could also be a mediating factor in the relationship between truancy and maladaptive use of the Internet. Adolescents engaging in online activities to an excessive degree could run the risk of disrupting their natural order of sleep. Evidence shows that increased sleep latency and decreased rapid eye movement sleep (REM-sleep) are significantly associated with excessive Internet use [65], while subjective insomnias and parasomnias are linked with truancy [66]. Sleep disorders have pronounced effects on daytime functioning and academic achievement. This could cause adolescents to become disinterested in school, thereby increasing the risk of school refusal and chronic absenteeism [66].

Poor nutrition and physical inactivity were shown to be significantly associated with PIU. Adolescents who spend longer hours online potentially navigate towards unhealthier foods. It is postulated that online gamers drink high-caffeinated energy drinks and eat high-sugar snacks to increase alertness for online gaming [67]. Subsequently, these factors could make online gamers more inclined to sedentary behaviors compared to non-gamers. Moreover, there is an extensive loyalty among gamers, particularly those who displace food, personal hygiene and physical activity, in order to continue with online games [68]. This could pose serious health-risks and may lead to severe psychosomatic symptoms.

\subsection{Multiple Risk-Behaviors}

Risk-behaviors were ascertained to be concurrent in nature, with $89.9 \%$ of adolescents in the PIU group reporting multiple risk-behaviors. These results are in line with Jessor's theory on problem behavior $[69,70]$. The problem behavior theory is a psychosocial model that attempts to explain behavioral outcomes in adolescents. It consists of three conceptual systems based on psychosocial components: personality system, perceived environmental system and behavior system. In the latter system, risk-behavior structures (e.g., alcohol use, tobacco use, delinquency and deviancy) tend to co-occur and cluster into a general 'risk-behavior syndrome' [71]. According to Jessor, these problem behaviors often stem from adolescents' assertion of independence from parents and societal influences.

Adolescents struggling for autonomy could, in part, account for the significant linear trend noted between hours online per day and multiple risk-behaviors. This trend was comparatively identical across all Internet user groups. These findings are highly relevant, as they suggest that excessive hours online in itself can increase the number of risk-behaviors for all adolescents and not only those diagnosed with PIU. Excessive hours online could also be a moderating factor in the relationship between PIU and risk-behaviors; however, further research exploring this relationship is necessitated.

\subsection{Gender Interactions}

The analysis on gender interactions showed that significant associations observed between risk-behaviors and PIU were evenly distributed between males and females. This is somewhat contradictory to previous research, which typically shows that PIU and risk-behaviors are specific to the male gender. This gender shift could be an indication that the gender gap for risk-behaviors may be narrowing among European adolescents.

From another perspective, the relationship between gender and risk-behaviors could be mediated by a third factor, such as psychopathology. In a large, gender-based study of adolescents $(n=56,086)$ aged 12-18 years, prevalence rates for PIU were estimated to be $2.8 \%$ among the total sample with significantly higher rates observed in males (3.6\%) compared to females (1.9\%) [72]. The respective study noted that females with emotional issues, such as subjective unhappiness or depressive symptoms, have a significantly higher PIU prevalence than males with similar emotional symptoms. Gender-based studies scrutinizing the effect of gender interactions on PIU are an essential prerequisite for the future direction of PIU research. 


\subsection{Griffiths' Components Model}

Griffiths' components model of addiction [30] hypothesizes that behavioral addictions (e.g., PIU) and substance-related addictions advance via similar biopsychosocial processes and share numerous physiognomies. The addiction criteria of the respective six core components in this model are (1) salience, (2) mood modification, (3) tolerance, (4) withdrawal, (5) conflict and (6) relapse. Kuss et al. [73] assessed the components model of addiction in two independent samples ( $\mathrm{n}=3105$ and $\mathrm{n}=2257$ ). Results showed that the components model of PIU fit the data very well in both samples.

In the present study, the YDQ measure was utilized to assess and detect adolescents with maladaptive and pathological risks related to their Internet use and online behaviors. As the YDQ measure comprises all six addiction criteria stipulated in Griffiths' components model, the validity of the outcomes reported in this study is supported by this theoretical framework.

\subsection{Strengths and Limitations}

The large, representative, cross-national sample is a major strength of this study. The homogenous methodology and standardized procedures utilized in all countries increases the validity, reliability and comparability of the data. To the extent of our knowledge, the geographic area in Europe was the largest ever used to conduct a study on PIU and risk-behaviors.

There are also some limitations of the study. Self-reported data are prone to recall and social desirability biases, which is likely to vary between countries and cultures. The cross-sectional design is unable to account for temporal relationships, thus causality could not be determined. In the GSHS measure, the subcategories of risk-taking actions only represent a part of sensation-seeking behaviors; thus, caution should be used when interpreting the results.

\section{Conclusions}

A significantly increasing prevalence rate across AIU, MIU and PIU groups was observed in all risk categories (substance use, sensation-seeking and lifestyle characteristics). Adolescents reporting poor sleeping habits and risk-taking actions showed the strongest associations with PIU, followed by tobacco use, poor nutrition and physical inactivity. The significant association observed between PIU and risk-behaviors, combined with a high rate of co-occurrence, underlines the importance of considering PIU when screening, treating or preventing high-risk behaviors in adolescents.

Among adolescents in the PIU group, $89.9 \%$ were characterized as having multiple risk-behaviors. Thus, efforts should target adolescents who excessively use the Internet, as a significant linear trend was observed between hours online per day and multiple risk-behaviors. This trend was similar across all Internet user groups indicating that excessive hours online in itself is an important factor for risk-behaviors. These findings need to be replicated and further explored before ascertaining their theoretical implications.

Acknowledgments: The SEYLE project was supported through Coordination Theme 1 (Health) of the European Union Seventh Framework Program (FP7), Grant Agreement No. HEALTH-F2-2009-223091. The authors were independent of the funders in all aspects of the study design, data analysis and writing of this manuscript. The Project Leader and Coordinator of the SEYLE project is Professor in Psychiatry and Suicidology Danuta Wasserman, Karolinska Institute (KI), Head of the National Centre for Suicide Research and Prevention of Mental Ill-Health and Suicide (NASP), at KI, Stockholm, Sweden. Other members of the Executive Committee are Senior Lecturer Vladimir Carli, National Centre for Suicide Research and Prevention of Mental Ill-Health (NASP), Karolinska Institute, Stockholm, Sweden; Christina W. H. Hoven and Anthropologist Camilla Wasserman, Department of Child and Adolescent Psychiatry, New York State Psychiatric Institute, Columbia University, New York, USA; and Marco Sarchiapone, Department of Health Sciences, University of Molise, Campobasso, Italy. The SEYLE Consortium comprises centers in 12 European countries. Site leaders for each respective center and country are: Danuta Wasserman (NASP, Karolinska Institute, Sweden, Coordinating Centre), Christian Haring (University for Medical Information Technology, Austria), Airi Varnik (Estonian Swedish Mental Health \& Suicidology Institute, Estonia), Jean-Pierre Kahn (University of Lorraine, Nancy, France), Romuald Brunner (University of Heidelberg, Germany), Judit Balazs (Vadaskert Child and Adolescent Psychiatric Hospital, Hungary), Paul Corcoran (National Suicide Research Foundation, Ireland), Alan Apter (Schneider Children's Medical Centre of Israel, Tel Aviv University, Tel Aviv, Israel), Marco Sarchiapone (University of Molise, Italy), 
Doina Cosman (Iuliu Hatieganu University of Medicine and Pharmacy, Romania), Vita Postuvan (University of Primorska, Slovenia) and Julio Bobes (University of Oviedo, Spain). Support for "Ethical Issues in Research with Minors and other Vulnerable Groups" was obtained by a grant from the Botnar Foundation, Basel, for Professor of Ethics, Stella Reiter-Theil, Psychiatric Clinic at Basel University, who served as the independent ethical consultant to the SEYLE project.

Author Contributions: Tony Durkee is the first and corresponding author who developed the study design, performed the statistical analyses and critically revised all phases of the manuscript. Vladimir Carli, Birgitta Floderus and Danuta Wasserman participated in the study design and made critical revisions to the manuscript. Camilla Wasserman, Christina W. Hoven, Michael Kaess and Peeter Värnik provided consultations and made critical revisions to the manuscript. Marco Sarchiapone, Alan Apter, Judit A. Balazs, Julio Bobes, Romuald Brunner, Paul Corcoran, Doina Cosman, Christian Haring, Jean-Pierre Kahn and Vita Postuvan are principal investigators for the SEYLE project in their respective countries and contributed to critical revisions to the manuscript. Bogdan Nemes and Pilar A. Saiz are project managers of the SEYLE project in their respective countries and participated in important revisions to the manuscript.

Conflicts of Interest: The authors declare no conflict of interest.

\section{Abbreviations}

The following abbreviations are used in this manuscript:

$\begin{array}{ll}\text { SEYLE } & \text { Saving and Empowering Young Lives in Europe } \\ \text { YRBSS } & \text { Youth Risk Behavior Surveillance System } \\ \text { GSHS } & \text { Global School-Based Student Health Survey } \\ \text { YDQ } & \text { Young's Diagnostic Questionnaire } \\ \text { GLMM } & \text { Generalized linear mixed models } \\ \text { ANOVA } & \text { One-way analysis of variance } \\ \text { PIU } & \text { Pathological Internet use } \\ \text { MIU } & \text { Maladaptive Internet use } \\ \text { AIU } & \text { Adaptive Internet use } \\ \text { CI } & \text { Confidence intervals } \\ \text { SEM } & \text { Standard error of the mean } \\ \text { M } & \text { Mean }\end{array}$

\section{References}

1. Moshman, D. Cognitive development beyond childhood. In Handbook of Child Psychology, 5th ed.; Kuhn, D., Damon, W., Siegler, R.S., Eds.; Wiley: New York, NY, USA, 1998; Volume 2, pp. 947-978.

2. Choudhury, S.; Blakemore, S.J.; Charman, T. Social cognitive development during adolescence. Soc. Cogn. Affect. Neurosci. 2006, 1, 165-174. [CrossRef] [PubMed]

3. Eccles, J.S.; Wigfield, A.; Byrnes, J. Cognitive development in adolescence. In Handbook of Psychology: Developmental Psychology; Lerner, R.M., Easterbrooks, M.A., Mistry, J., Eds.; Wiley: Hoboken, NJ, USA, 2003; Volume 6, pp. 325-350.

4. Subrahmanyam, K.; Greenfield, P.; Kraut, R.; Gross, E. The impact of computer use on children's and adolescents' development. J. Appl. Dev. Psychol. 2001, 22, 7-30. [CrossRef]

5. Ellison, N.B.; Steinfield, C.; Lampe, C. The benefits of Facebook "friends": Social capital and college students' use of online social network sites. J. Comput. Med. Commun. 2007, 12, 1143-1168. [CrossRef]

6. Steinfield, C.; Ellison, N.B.; Lampe, C. Social capital, self-esteem, and use of online social network sites: A longitudinal analysis. J. Appl. Dev. Psychol. 2008, 29, 434-445. [CrossRef]

7. Tapscott, D. Growing up Digital: The Rise of the Net Generation; McGraw-Hill Education: New York, NY, USA, 2008; p. 384.

8. Kraut, R.; Patterson, M.; Lundmark, V.; Kiesler, S.; Mukopadhyay, T.; Scherlis, W. Internet paradox. A social technology that reduces social involvement and psychological well-being? Am. Psychol. 1998, 53, 1017-1031. [CrossRef] [PubMed] 
9. Kraut, R.; Kiesler, S.; Boneva, B.; Cummings, J.; Helgeson, V.; Crawford, A. Internet paradox revisited. J. Soc. Issues 2002, 58, 49-74. [CrossRef]

10. Nie, N.H.; Hillygus, D.S.; Erbring, L. Internet use, interpersonal relations, and sociability: A time diary study. In The Internet in Everyday Life; Wellman, B., Haythornthwaite, C., Eds.; Blackwell Publishers Ltd.: Oxford, UK, 2002; pp. 213-243.

11. Nalwa, K.; Anand, A.P. Internet addiction in students: A cause of concern. Cyberpsychol. Behav. 2003, 6, 653-656. [CrossRef] [PubMed]

12. Akhter, N. Relationship between internet addiction and academic performance among university undergraduates. Edu. Res. Rev. 2013, 8, 1793.

13. Gür, K.; Yurt, S.; Bulduk, S.; Atagöz, S. Internet addiction and physical and psychosocial behavior problems among rural secondary school students. Nurs. Health Sci. 2015, 17, 331-338. [CrossRef] [PubMed]

14. Peltzer, K.; Pengpid, S.; Apidechkul, T. Heavy internet use and its associations with health risk and health-promoting behaviours among Thai university students. Int. J. Adolesc. Med. Health 2014, 26, 187-194. [CrossRef] [PubMed]

15. Punamaki, R.L.; Wallenius, M.; Nygard, C.H.; Saarni, L.; Rimpela, A. Use of information and communication technology (ICT) and perceived health in adolescence: The role of sleeping habits and waking-time tiredness. J. Adolesc. 2007, 30, 569-585. [CrossRef] [PubMed]

16. Straker, L.; Pollock, C.; Maslen, B. Principles for the wise use of computers by children. Ergonomics 2009, 52, 1386-1401. [CrossRef] [PubMed]

17. Shaw, M.; Black, D.W. Internet addiction: Definition, assessment, epidemiology and clinical management. CNS Drugs 2008, 22, 353-365. [CrossRef] [PubMed]

18. Young, K. Internet addiction: The emergence of a new clinical disorder. CyberPsychol. Behav. 1998, 1, $237-244$. [CrossRef]

19. American Psychiatric Association (APA). Diagnostic and statistical manual of mental disorders. Available online: http:/ /www.dsm5.org (accessed on 2 February 2016).

20. Petry, N.M.; O'Brien, C.P. Internet gaming disorder and the DSM-5. Addiction 2013, 108, 1186-1187. [CrossRef] [PubMed]

21. Sussman, S.; Lisha, N.; Griffiths, M. Prevalence of the addictions: A problem of the majority or the minority? Eval. Health Prof. 2011, 34, 3-56. [CrossRef] [PubMed]

22. Lee, H.W.; Choi, J.S.; Shin, Y.C.; Lee, J.Y.; Jung, H.Y.; Kwon, J.S. Impulsivity in internet addiction: A comparison with pathological gambling. Cyberpsychol. Behav. Soc. Netw. 2012, 15, 373-377. [CrossRef] [PubMed]

23. Tonioni, F.; Mazza, M.; Autullo, G.; Cappelluti, R.; Catalano, V.; Marano, G.; Fiumana, V.; Moschetti, C.; Alimonti, F.; Luciani, M. Is internet addiction a psychopathological condition distinct from pathological gambling? Addict. Behav. 2014, 39, 1052-1056. [CrossRef] [PubMed]

24. Sajeev Kumar, P.; Prasad, N.; Raj, Z.; Abraham, A. Internet addiction and substance use disorders in adolescent students-a cross sectional study. J. Int. Med. Dent. 2015, 2, 172-179.

25. Brezing, C.; Derevensky, J.L.; Potenza, M.N. Non-substance-addictive behaviors in youth: Pathological gambling and problematic internet use. Child Adoles. Psychiatr. Clin. N. Am. 2010, 19, 625-641. [CrossRef] [PubMed]

26. Goldstein, R.Z.; Volkow, N.D. Dysfunction of the prefrontal cortex in addiction: Neuroimaging findings and clinical implications. Nat. Rev. Neurosci. 2011, 12, 652-669. [CrossRef] [PubMed]

27. Montag, C.; Kirsch, P.; Sauer, C.; Markett, S.; Reuter, M. The role of the chrna4 gene in internet addiction: A case-control study. J. Addict. Med. 2012, 6, 191-195. [CrossRef] [PubMed]

28. Kormas, G.; Critselis, E.; Janikian, M.; Kafetzis, D.; Tsitsika, A. Risk factors and psychosocial characteristics of potential problematic and problematic internet use among adolescents: A cross-sectional study. BMC Public Health 2011, 11, 595. [CrossRef] [PubMed]

29. Zhou, Y.; Lin, F.-C.; Du, Y.-S.; Zhao, Z.-M.; Xu, J.-R.; Lei, H. Gray matter abnormalities in internet addiction: A voxel-based morphometry study. Eur. J. Radiol. 2011, 79, 92-95. [CrossRef] [PubMed]

30. Griffiths, M. A "components" model of addiction within a biopsychosocial framework. J. Subst. Use 2005, 10, 191-197. [CrossRef] 
31. Cheng, C.; Li, A.Y. Internet addiction prevalence and quality of (real) life: A meta-analysis of 31 nations across seven world regions. Cyberpsychol. Behav. Soc. Netw. 2014, 17, 755-760. [CrossRef] [PubMed]

32. Blinka, L.; Škařupová, K.; Ševčíková, A.; Wölfling, K.; Müller, K.W.; Dreier, M. Excessive internet use in European adolescents: What determines differences in severity? Int. J. Public Health 2015, 60, 249-256. [CrossRef] [PubMed]

33. Tsitsika, A.; Janikian, M.; Schoenmakers, T.M.; Tzavela, E.C.; Ólafsson, K.; Wójcik, S.; Florian Macarie, G.; Tzavara, C.; Richardson, C. Internet addictive behavior in adolescence: A cross-sectional study in seven European countries. Cyberpsychol. Behav. Soc. Netw. 2014, 17, 528-535. [CrossRef] [PubMed]

34. Durkee, T.; Kaess, M.; Carli, V.; Parzer, P.; Wasserman, C.; Floderus, B.; Apter, A.; Balazs, J.; Barzilay, S.; Bobes, J.; et al. Prevalence of pathological internet use among adolescents in Europe: Demographic and social factors. Addiction 2012, 107, 2210-2222. [CrossRef] [PubMed]

35. Kuss, D.J.; Griffiths, M.D.; Karila, L.; Billieux, J. Internet addiction: A systematic review of epidemiological research for the last decade. Curr. Pharm. Des. 2014, 20, 4026-4052. [CrossRef] [PubMed]

36. Carli, V.; Durkee, T.; Wasserman, D.; Hadlaczky, G.; Despalins, R.; Kramarz, E.; Wasserman, C.; Sarchiapone, M.; Hoven, C.W.; Brunner, R.; et al. The association between pathological internet use and comorbid psychopathology: A systematic review. Psychopathology 2013, 46, 1-13. [CrossRef] [PubMed]

37. Ho, R.C.; Zhang, M.W.; Tsang, T.Y.; Toh, A.H.; Pan, F.; Lu, Y.; Cheng, C.; Yip, P.S.; Lam, L.T.; Lai, C.-M.; et al. The association between internet addiction and psychiatric co-morbidity: A meta-analysis. BMC Psychiatry 2014, 14, 1-10. [CrossRef] [PubMed]

38. Kaess, M.; Durkee, T.; Brunner, R.; Carli, V.; Parzer, P.; Wasserman, C.; Sarchiapone, M.; Hoven, C.; Apter, A.; Balazs, J.; et al. Pathological internet use among European adolescents: Psychopathology and self-destructive behaviours. Eur. Child Adolesc. Psychiatry 2014, 23, 1093-1102. [CrossRef] [PubMed]

39. Pontes, H.M.; Kuss, D.J.; Griffiths, M.D. The clinical psychology of internet addiction: A review of its conceptualization, prevalence, neuronal processes, and implications for treatment. Neurosci. Neuroeconomics 2015, 4, 11-23.

40. Kipping, R.R.; Campbell, R.M.; MacArthur, G.J.; Gunnell, D.J.; Hickman, M. Multiple risk behaviour in adolescence. J. Public Health 2012, 34, i1-i2. [CrossRef] [PubMed]

41. Dodd, L.J.; Al-Nakeeb, Y.; Nevill, A.; Forshaw, M.J. Lifestyle risk factors of students: A cluster analytical approach. Prev. Med. 2010, 51, 73-77. [CrossRef] [PubMed]

42. Berk, M.; Sarris, J.; Coulson, C.; Jacka, F. Lifestyle management of unipolar depression. Acta Psychiatr. Scand. 2013, 127, 38-54. [CrossRef] [PubMed]

43. Prochaska, J.J.; Spring, B.; Nigg, C.R. Multiple health behavior change research: An introduction and overview. Prev. Med. 2008, 46, 181-188. [CrossRef] [PubMed]

44. Carli, V.; Hoven, C.W.; Wasserman, C.; Chiesa, F.; Guffanti, G.; Sarchiapone, M.; Apter, A.; Balazs, J.; Brunner, R.; Corcoran, P. A newly identified group of adolescents at "invisible" risk for psychopathology and suicidal behavior: Findings from the SEYLE study. World Psychiatry 2014, 13, 78-86. [CrossRef] [PubMed]

45. Kann, L.; Kinchen, S.; Shanklin, S.L.; Flint, K.H.; Kawkins, J.; Harris, W.A.; Lowry, R.; Olsen, E.; McManus, T.; Chyen, D. Youth risk behavior surveillance-United States, 2013. MMWR Surveill. Summ. 2014, 63, 1-168.

46. Wasserman, D.; Carli, V.; Wasserman, C.; Apter, A.; Balazs, J.; Bobes, J.; Bracale, R.; Brunner, R.; Bursztein-Lipsicas, C.; Corcoran, P.; et al. Saving and empowering young lives in Europe (SEYLE): A randomized controlled trial. BMC Public Health 2010, 10, 192. [CrossRef] [PubMed]

47. Carli, V.; Wasserman, C.; Wasserman, D.; Sarchiapone, M.; Apter, A.; Balazs, J.; Bobes, J.; Brunner, R.; Corcoran, P.; Cosman, D. The saving and empowering young lives in Europe (SEYLE) randomized controlled trial (RCT): Methodological issues and participant characteristics. BMC Public Health 2013, 13, 479. [CrossRef] [PubMed]

48. Young, K.S. Caught in the Net: How to Recognize the Signs of Internet Addiction-and a Winning Strategy for Recovery; J. Wiley: New York, NY, USA, 1998; p. 248.

49. Dowling, N.A.; Quirk, K.L. Screening for internet dependence: Do the proposed diagnostic criteria differentiate normal from dependent internet use? Cyberpsychol. Behav. 2009, 12, 21-27. [CrossRef] [PubMed] 
50. Li, W.; O’Brien, J.E.; Snyder, S.M.; Howard, M.O. Diagnostic criteria for problematic internet use among U.S. University students: A mixed-methods evaluation. PLoS ONE 2016, 11, e0145981. [CrossRef] [PubMed]

51. Pontes, H.M.; Király, O.; Demetrovics, Z.; Griffiths, M.D. The conceptualisation and measurement of dsm-5 internet gaming disorder: The development of the IGD-20 test. PLoS ONE 2014, 9, e110137. [CrossRef] [PubMed]

52. World Health Organization (WHO). Global School-Based Student Health Survey (GSHS). Available online: http://www.who.int/chp/gshs/en/ (accessed on 12 December 2015).

53. Choi, K.; Son, H.; Park, M.; Han, J.; Kim, K.; Lee, B.; Gwak, H. Internet overuse and excessive daytime sleepiness in adolescents. Psychiatry Clin. Neurosci. 2009, 63, 455-462. [CrossRef] [PubMed]

54. Evren, C.; Dalbudak, E.; Evren, B.; Demirci, A.C. High risk of internet addiction and its relationship with lifetime substance use, psychological and behavioral problems among 10th grade adolescents. Psychiatria Danub. 2014, 26, 330-339.

55. International telecommunication union (ITU). ICT Facts and Figures. Available online: http:/ /www.itu.int/en (accessed on 8 August 2015).

56. De La Haye, K.; D'Amico, E.J.; Miles, J.N.; Ewing, B.; Tucker, J.S. Covariance among multiple health risk behaviors in adolescents. PLOS ONE 2014, 9, e98141.

57. Cao, F.; Su, L.; Liu, T.; Gao, X. The relationship between impulsivity and internet addiction in a sample of Chinese adolescents. Eur. Psychiatry: J. Assoc. Eur. Psychiatr. 2007, 22, 466-471. [CrossRef] [PubMed]

58. Slater, M.D. Alienation, aggression, and sensation seeking as predictors of adolescent use of violent film, computer, and website content. J. Commun. 2003, 53, 105-121. [CrossRef]

59. Kim, H.K.; Davis, K.E. Toward a comprehensive theory of problematic internet use: Evaluating the role of self-esteem, anxiety, flow, and the self-rated importance of internet activities. Comput. Hum. Behav. 2009, 25, 490-500. [CrossRef]

60. Charlton, J.P.; Danforth, I.D. Distinguishing addiction and high engagement in the context of online game playing. Comput. Hum. Behav. 2007, 23, 1531-1548. [CrossRef]

61. Kuss, D.J.; Griffiths, M.D. Online social networking and addiction-A review of the psychological literature. Int. J. Environ. Res. Public Health 2011, 8, 3528-3552. [CrossRef] [PubMed]

62. Meena, P.S.; Mittal, P.K.; Solanki, R.K. Problematic use of social networking sites among urban school going teenagers. Ind. Psychiatry J. 2012, 21, 94. [CrossRef] [PubMed]

63. Li, W.; O'Brien, J.E.; Snyder, S.M.; Howard, M.O. Characteristics of internet addiction/pathological internet use in U.S. University students: A qualitative-method investigation. PLoS ONE 2015, 10, e0117372. [CrossRef] [PubMed]

64. Lam, L. Internet gaming addiction, problematic use of the internet, and sleep problems: A systematic review. Curr. Psychiatry Rep. 2014, 16, 1-9. [CrossRef] [PubMed]

65. Cain, N.; Gradisar, M. Electronic media use and sleep in school-aged children and adolescents: A review. Sleep Med. 2010, 11, 735-742. [CrossRef] [PubMed]

66. Hochadel, J.; Frolich, J.; Wiater, A.; Lehmkuhl, G.; Fricke-Oerkermann, L. Prevalence of sleep problems and relationship between sleep problems and school refusal behavior in school-aged children in children's and parents' ratings. Psychopathology 2014, 47, 119-126. [CrossRef] [PubMed]

67. Lin, S.S.J.; Tsai, C.C. Sensation seeking and internet dependence of Taiwanese high school adolescents. Comput. Hum. Behav. 2002, 18, 411-426. [CrossRef]

68. Hsi-Peng, L.; Shu-ming, W. The role of internet addiction in online game loyalty: An exploratory study. Internet Res. 2008, 18, 499-519.

69. Jessor, R.; Jessor, S.L. Problem Behavior and Psychosocial Development: A Longitudinal Study of Youth; Academic Press: Cambridge, MA, USA, 1977; p. 281.

70. Jessor, R. Problem-behavior theory, psychosocial development, and adolescent problem drinking. Br. J. Addict. 1987, 82, 331-342. [CrossRef] [PubMed]

71. Williams, J.H.; Ayers, C.D.; Abbott, R.D.; Hawkins, J.D.; Catalano, R.F. Structural equivalence of involvement in problem behavior by adolescents across racial groups using multiple group confirmatory factor analysis. Soc. Work Res. 1996, 20, 168-177. 
72. Ha, Y.-M.; Hwang, W.J. Gender differences in internet addiction associated with psychological health indicators among adolescents using a national web-based survey. Int. J. Ment. Health Addict. 2014, 12, 660-669. [CrossRef]

73. Kuss, D.J.; Shorter, G.W.; van Rooij, A.J.; Griffiths, M.D.; Schoenmakers, T.M. Assessing internet addiction using the parsimonious internet addiction components model-A preliminary study. Int. J. Ment. Health Addict. 2014, 12, 351-366. [CrossRef]

(C) 2016 by the authors; licensee MDPI, Basel, Switzerland. This article is an open access article distributed under the terms and conditions of the Creative Commons by Attribution (CC-BY) license (http:/ / creativecommons.org/licenses/by/4.0/). 